\title{
Physical Activity in Patients with Inflammatory Bowel Disease: A Cross-Sectional Study
}

\author{
Georgina Fagan ${ }^{a}$ Hamish Osborne ${ }^{a}$ Michael Schultz ${ }^{a, b}$ \\ aDepartment of Medicine, Dunedin School of Medicine, University of Otago, Dunedin, New Zealand; \\ ${ }^{\mathrm{b}}$ Gastroenterology Department, Southern District Health Board, Dunedin, New Zealand
}

\section{Keywords}

Inflammatory bowel disease · Physical activity · Quality of life $\cdot$ Crohn's disease $\cdot$ Ulcerative colitis

\begin{abstract}
Introduction and Objective: Despite the known benefits of physical activity in the management of chronic diseases, the use of exercise as a treatment is relatively underemployed, with many patients reporting their disease to be a barrier. This study aimed to assess physical activity levels, attitudes, and barriers towards exercise in a cohort of patients with inflammatory bowel disease (IBD). In addition, this study aimed to assess possible relationships between physical well-being, psychological well-being, and sociodemographic factors. Methods: 306 patients $>18$ years with diagnosed IBD in Dunedin, New Zealand, were approached to participate in this study. Participants who consented completed questionnaires regarding exercise preferences and attitudes, physical activity levels, and psychological well-being. Results: Seventy-seven patients participated in the study $(77 / 308=25 \%)$. Sixty-six percent of participants met physical activity guidelines and the median metabolic equivalent minutes/week of 1,027.5 (505.5-2,339.5). Walking was the most preferred activity
\end{abstract}

karger@karger.com www.karger.com/iid

Karger $\stackrel{\text { ' }}{5}$

GOPEN ACCESS
(C) 2021 The Author(s)

Published by S. Karger AG, Basel

This is an Open Access article licensed under the Creative Commons Attribution-NonCommercial-4.0 International License (CC BY-NC) (http://www.karger.com/Services/OpenAccessLicense), applicable to the online version of the article only. Usage and distribution for commercial purposes requires written permission.
(30\%) followed by water-based exercise (20\%). Two-thirds of participants reported their disease limited the amount of physical activity undertaken. Common barriers to participating in physical activity included fatigue (34\%) and abdominal pain (20\%). Patients with active disease reported higher levels of barriers than those in remission ( 80 vs. $54 \%, p=0.018$ ). Higher physical activity levels were correlated with lower levels of fatigue ( $p=0.022-0.046)$. Conclusions: While patients with IBD in Dunedin, New Zealand, are physically active, reducing real and perceived barriers is crucial to further increase physical activity in patients with IBD who are in remission or with active disease. (c) 2021 The Author(s)

Published by S. Karger AG, Basel

\section{Introduction}

Inflammatory bowel diseases (IBD), which incorporate Crohn's disease (CD), ulcerative colitis (UC), and inflammatory bowel disease-unclassified (IBD-U), are chronic, systemic, and undulating inflammatory conditions affecting primarily the gastrointestinal tract of patients [1]. The global incidence of IBD has been rising steadily in recent years $[2,3]$, and a study conducted in 
southern New Zealand by Coppell et al. [4] showed a peak incidence of IBD of $29.8 / 100,000$ people. While the exact mechanism and cause of IBD are unknown, it is thought to incorporate 4 key elements: genetic predisposition, environmental triggers, abnormal immune response, and gut microbiome changes $[1,5]$.

Many patients with IBD suffer from significant fatigue [6], depression and anxiety [7], and a reduced health-related quality of life (HRQoL) [7] because of the impact the symptoms of the disease can have on their life. The level of their psychological well-being does not always correlate with the severity of their disease. For example, Aluzaite et al. [8] reported that while $83 \%$ of patients with active IBD experience fatigue, up to $44 \%$ of patients whose disease is in remission can also suffer from significant fatigue. It is clear that just treating IBD with current medical management is ineffective at improving patient's psychological well-being, and this is directing researchers to investigate other options such as increasing physical activity [9].

Physical activity is defined as any bodily movement produced by skeletal muscles resulting in energy expenditure [10]. Regular physical activity has been shown to significantly improve health in the general "healthy" population in numerous ways including decreasing blood pressure, body weight, and bone loss, as well as improving sleep quality and immune response [11]. Exercise is well known to improve psychological well-being, including reducing depression and anxiety levels, fatigue, and stress [12]. In patients with IBD, habitual exercise has been found to increase the time spent in remission [13], improve gastrointestinal permeability [14], and improve HRQoL [15].

While the numerous health benefits from regular exercise are well known, the level of physical activity engagement worldwide is poor, with only $50 \%$ of the general population meeting the current physical activity guideline of 150 MET-mins/week $[16,17]$. The majority of studies worldwide report that patients with IBD are also not meeting the recommended physical activity levels [18-21], but the level of physical activity in patients with IBD in New Zealand is currently unknown.

Therefore, the primary objective of the study was to investigate current physical activity levels in a cohort of participants with IBD in Dunedin, New Zealand. Secondary objectives included assessing participants' attitudes and preference towards exercise and investigating any relationship between physical activity levels and quality of life, depression, anxiety, fatigue, and disease activity.

\section{Materials and Methods}

\section{Participants}

Potential participants were identified through the Dunedin Hospital gastroenterology outpatient clinics (Dunedin, New Zealand) or through the EpiSoft ${ }^{\circledR}$ database (Sydney, Australia) and approached via an invitation letter between May and August 2017 as part of a study investigating compliance to a personalized exercise programme [22]. Patients older than 18 years of age, with a documented diagnosis of IBD, whose disease was in remission or with mild-moderate activity were considered eligible for the study. Patients were excluded from the study if they had severe active IBD, were currently pregnant, had undergone surgery in the past month, or who had a physical disability or other medical condition that would limit their ability to participate in physical activity. A STROBE flow diagram for this study is shown in Figure 1.

\section{Sociodemographic and Clinical Data Collection}

Participants who consented were asked to complete several written questionnaires. Standard choice questions were used to assess basic demographic information including age, gender, ethnicity, weight and height (for body mass index [BMI] calculations), disease type (CD, UC, and IBD-U), time since diagnosis, previous surgical history, current medication use (including over-the-counter medication/supplements), and smoking status (current/never/ ex-smoker). Harvey-Bradshaw Index (HBI) for those with CD and the Simple Clinical Colitis Activity Index (SCCAI) for those with UC were used to assess disease activity levels. A HBI score of $<5$ indicated remission in CD [23], and a SCCAI score of $\leq 3$ indicated remission in UC [24]. Current laboratory blood results were taken at the time of questionnaire completion and included complete blood count, C-reactive protein (CRP), and ferritin levels. Faecal calprotectin (FC) levels were also assessed.

\section{Study Questionnaires}

The International Physical Activity Questionnaire (IPAQ)short form was used to assess participants' level of physical activity [25]. It assesses the time spent participating in vigorous exercise, moderate-intensity exercise, or walking, each for $>10 \mathrm{~min}$ at a time, in the last 7 days. Scores for each category are measured as metabolic equivalent minutes/week (MET-min/week). Scores from the IPAQ can be grouped into 3 categories: low physical activity (score $<600 \mathrm{MET}$-mins/week), moderate physical activity (approximate score between 600 and 3,000 MET-mins/week), and high physical activity (approximate score >3,000 MET-mins/ week).

Exercise preferences and attitudes towards exercise were assessed through a novel questionnaire designed by the authors, requiring participants to rank chosen exercise types (such as walking and swimming) and identify any barriers to physical activity they had experienced. Depression and anxiety levels were assessed with the Hospital Anxiety and Depression Scale (HADS) [26]. The HADS contains 7 questions measuring anxiety levels and 7 questions assessing depression levels with each question scoring $0-3$ points. Total scores for anxiety and depression are therefore out of 21 with higher scores indicating more severe levels.

Fatigue was assessed through both the Inflammatory Bowel Disease Fatigue Patient Self-Assessment Scale (IBD-F) and the Multidimensional Fatigue Inventory (MFI). The IBD-F is the first scale to be specifically designed for patients with IBD [27] and con-
62

Inflamm Intest Dis 2021;6:61-69 DOI: $10.1159 / 000511212$
Fagan/Osborne/Schultz 
Fig. 1. STROBE flow diagram of the study. IBD, inflammatory bowel disease.

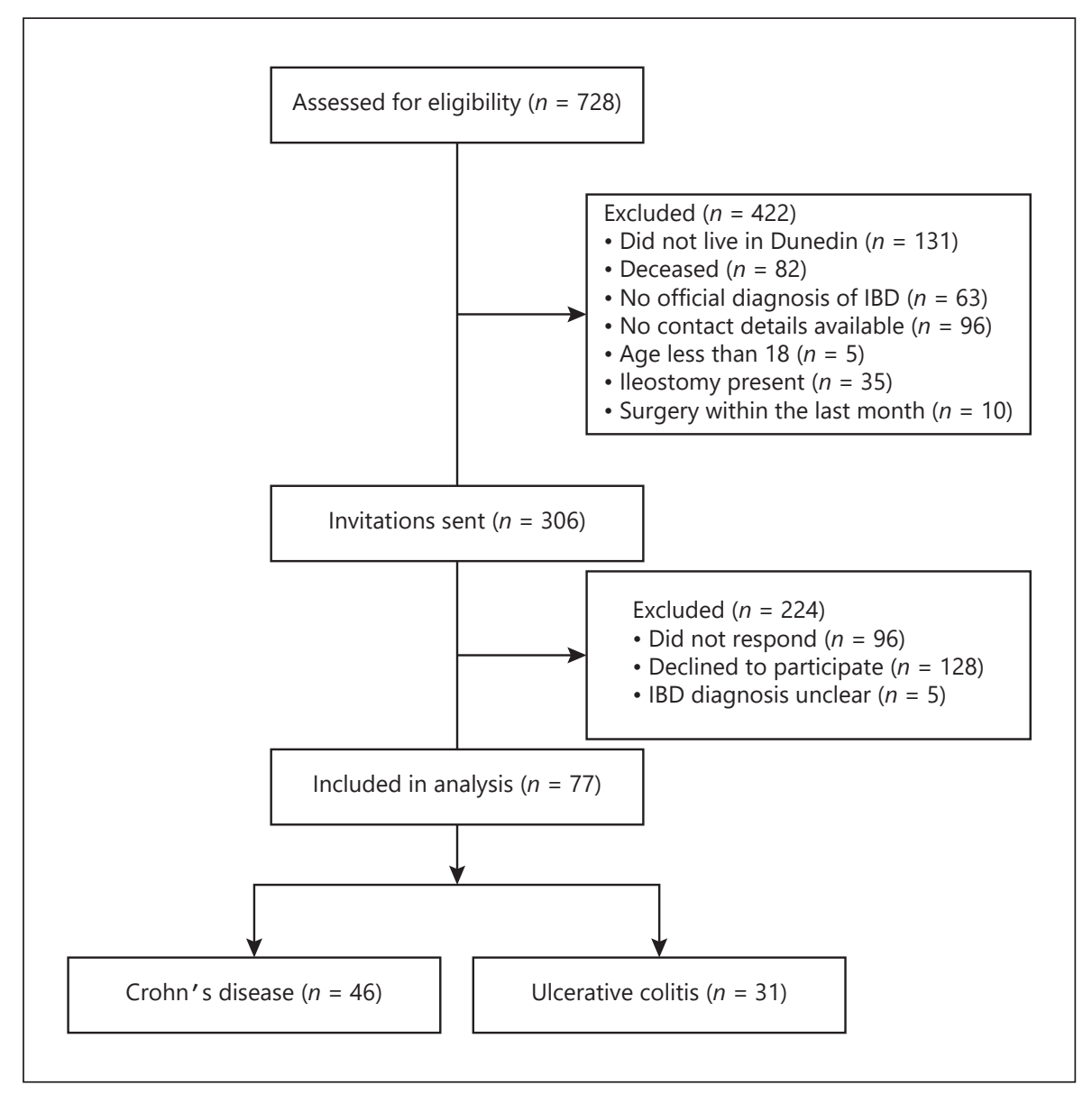

sists of 3 sections: the first section contains 5 questions and assesses the frequency and severity of fatigue experienced, the second section composed of 30 questions and measures the impact of fatigue on patients' life, and the third one contains unscored openended questions on possible factors influencing fatigue.

The MFI is the most widely used fatigue questionnaire in studies with IBD patients $[6,28]$. It contains 20 questions that assess 5 different subscales (physical fatigue, mental fatigue, general fatigue, reduced activity, and reduced motivation) and provides details about impact and severity of the fatigue experienced. Each question asks the patient to determine how much they agree with the statement on a 1-5 Likert scale. Each dimension of the scale is used independently. Higher scores indicate higher levels of fatigue for both questionnaires.

HRQoL was assessed through the Inflammatory Bowel Disease Quality of Life Questionnaire (IBDQ) [29]. The IBDQ, which is the only specifically designed HRQoL questionnaire for IBD populations, consists of 32 questions. Higher scores represent better HRQoL. The IBDQ can be further subdivided into 4 dimensions: bowel symptoms, systemic symptoms, emotional well-being, and social functioning.

\section{Ethical Consideration}

Participants provided written consent to participate in this study. This study was approved by the Northern B Health and Dis- ability Ethics Committee, New Zealand, Reference No. 17/NTB/44. The trial was registered on ANZCTR, No.ACTRN12617000526370, Universal Trial No. U1111-1192-2859.

\section{Statistical Analysis}

Statistical analyses were performed using SPSS (v. 24, IBM Corp., Armonk, NY, USA). Sample size was not calculated for this study as it was part of a small pilot study [22].

Continuous data are presented as mean \pm SD unless otherwise stated. Pearson's correlation coefficients were used to investigate correlations between physical activity, baseline demographics, and psychological well-being. Multiple linear regression was carried out including any variables associated with total physical activity levels $(p>0.10)$. All regression models were fitted after testing assumptions were met for the variables involved. A two-sided $p$ value of $<0.05$ was used to show statistically significant results.

\section{Results}

\section{Baseline Demographics}

A total of 308 patients were approached for inclusion in the current study. Of those, 77 patients consented to 
participate (Fig. 1). Sociodemographic data of those who consented to participate in the study are included in Table 1.

Fifty-three percent of participants were female. The majority (90\%) were of New Zealand European ethnicity, and the mean age was $45.5 \pm 16.4$ years. Just over half of participants had CD (60\%), the mean time since diagnosis was on average 15 years, and $51 \%$ of all participants had disease in remission (Table 1).

\section{Physical Activity Levels}

Sixty-six percent of participants met physical activity guidelines of 5, 30-min moderate-intensity exercise sessions a week. Half of the participants were in the moderate physical activity category, and the mean total METmins/week was 1,613.0 (95\% CI: 1,221.9-2,004.1) (Table 1). Patients with CD participated in on average 834.9 MET-mins more physical activity per week than patients with UC (95\% CI: $140.4-1,529.3 ; p=0.019)$. There was no difference in physical activity between patients with active disease and disease in remission (mean difference -329.3 ; 95\% CI: $-1,125.0$ to 466.2 ; $p=0.412$ ) (Fig. 2).

For patients with $\mathrm{CD}$, in univariate analysis, higher levels of vigorous physical activity were associated with lower fatigue (IBD-F section 1: $r=-0.306, p=0.043$; MFI-physical: $r=-0.376, p=0.012$ ) and higher HRQoL (IBDQ-bowel: $r=0.322, p=0.035$; IBDQ-systemic: $r=$ $0.380, p=0.012$ ). Males were more likely to participate in higher levels of vigorous physical activity than females, a difference of 548.9 MET-mins (95\% CI: 91.1$1,006.7 ; p=0.020$ ) (see online suppl. Table 1; see www. karger.com/doi/10.1159/000511212 for all online suppl. material).

For patients with UC, in univariate analysis, lower total physical activity levels were associated with higher CRP levels $(r=-0.399, p=0.029)$ and lower HRQoL $(r=-0.407, p=0.023)$. There were no other variables correlated with physical activity levels in patients with UC. Full univariate analysis is available in online suppl. Table 2.

For CD patients, the multiple regression model did not significantly predict IPAQ total physical activity scores $(F[5,28]=1.632, p=0.184)$ and explained $9 \%$ of the variance in HRQoL scores. No variables included in the regression were independently associated with IPAQ total physical activity scores (Table 2).

In patients with $\mathrm{UC}$, the multiple regression model statistically significantly predicted IPAQ total physical activity scores $(F[2,27]=7.024, p=0.003)$, with the model
Table 1. Baseline participant characteristics by disease type

\begin{tabular}{|c|c|c|c|}
\hline Variable & $\begin{array}{l}\text { Crohn's } \\
\text { disease } \\
(n=46)\end{array}$ & $\begin{array}{l}\text { Ulcerative } \\
\text { colitis } \\
(n=31)\end{array}$ & $\begin{array}{l}\text { Overall } \\
(n=77)\end{array}$ \\
\hline \multicolumn{4}{|l|}{ Gender, $n(\%)$} \\
\hline Male & $23(50)$ & $13(42)$ & $36(47)$ \\
\hline Female & $23(50)$ & $18(58)$ & $41(53)$ \\
\hline Age, years & $44.0 \pm 15.2$ & $47.7 \pm 18.1$ & $45.5 \pm 16.4$ \\
\hline \multicolumn{4}{|l|}{ Ethnicity, $n(\%)$} \\
\hline New Zealand European & $41(89)$ & $28(90)$ & $69(90)$ \\
\hline Māori & $1(2)$ & $1(3)$ & $2(3)$ \\
\hline Others & $4(9)$ & $2(7)$ & $6(8)$ \\
\hline Time since diagnosis, years & $14.4 \pm 13.3$ & $16.7 \pm 13.5$ & $15.4 \pm 13.3$ \\
\hline $\mathrm{BMI}, \mathrm{kg} / \mathrm{m}^{2}$ & $27.1 \pm 4.7$ & $29.7 \pm 8.7$ & $28.2 \pm 6.7$ \\
\hline Currently on medication, $n(\%)$ & $39(85)$ & $23(74)$ & $62(81)$ \\
\hline \multicolumn{4}{|l|}{ Current medications, $n(\%)$} \\
\hline Aminosalicylates & $12(26)$ & $19(61)$ & $31(40)$ \\
\hline Biologic therapy & $10(22)$ & $3(10)$ & $13(17)$ \\
\hline Immunomodulators & $23(50)$ & $5(16)$ & $28(36)$ \\
\hline Corticosteroids & $2(4)$ & $2(7)$ & $4(5)$ \\
\hline \multicolumn{4}{|l|}{ Disease activity, mean \pm SD } \\
\hline HBI & $4.3 \pm 3.3$ & & \\
\hline SCCAI & & $3.8 \pm 2.6$ & \\
\hline Patients in remission, $n(\%)$ & $24(53)$ & $17(55)$ & $41(53)$ \\
\hline Previous surgery, $n(\%)$ & $25(56)$ & 0 & $26(34)$ \\
\hline Family with IBD, $n(\%)$ & $12(26)$ & $11(36)$ & $23(30)$ \\
\hline Following a specific diet, $n(\%)$ & $8(17)$ & $12(39)$ & $20(26)$ \\
\hline Taking supplements, $n(\%)$ & $13(28)$ & $11(36)$ & $24(31)$ \\
\hline Ferritin, $\mu \mathrm{g} / \mathrm{L}$ & $126 \pm 142$ & $121 \pm 150$ & $111 \pm 120$ \\
\hline $\mathrm{CRP}, \mathrm{mg} / \mathrm{L}$ & $5 \pm 8$ & $4 \pm 8$ & $4 \pm 6$ \\
\hline $\mathrm{FC}, \mu \mathrm{g} / \mathrm{g}$ & $286 \pm 425$ & $281 \pm 384$ & $291 \pm 402$ \\
\hline \multicolumn{4}{|c|}{ Physical activity level, IPAQ categories, $n(\%)$} \\
\hline Low & $14(30)$ & $11(36)$ & $25(33)$ \\
\hline Moderate & $21(46)$ & $19(61)$ & $40(52)$ \\
\hline High & $11(24)$ & $1(3)$ & $12(16)$ \\
\hline
\end{tabular}

Data are presented as mean $\pm \mathrm{SD}$, unless otherwise stated. BMI, body mass index; HBI, Harvey-Bradshaw Index; SCCAI, Simple Clinical Colitis Activity Index; CRP, C-reactive protein; FC, faecal calprotectin; MET-mins/week, metabolic equivalent minutes per week; IPAQ, International Physical Activity Questionnaire.

explaining $30 \%$ of the variance in physical activity. Both CRP levels and IBDQ-B score were independently associated with IPAQ total scores, $p=0.013$ and $p=0.008$, respectively. Regression coefficients and standard errors for both CD and UC can be found in Table 2 .

\section{Attitudes and Preferences to Exercise}

Sixty-six percent of participants reported that their IBD has limited the amount of physical activity that they could participate in at some point. Patients with active disease reported higher levels of barriers when compared
64

Inflamm Intest Dis 2021;6:61-69 DOI: $10.1159 / 000511212$
Fagan/Osborne/Schultz 
Table 2. Multiple linear regression model for physical activity levels (IPAQ - total category) for both Crohn's disease and ulcerative colitis participants

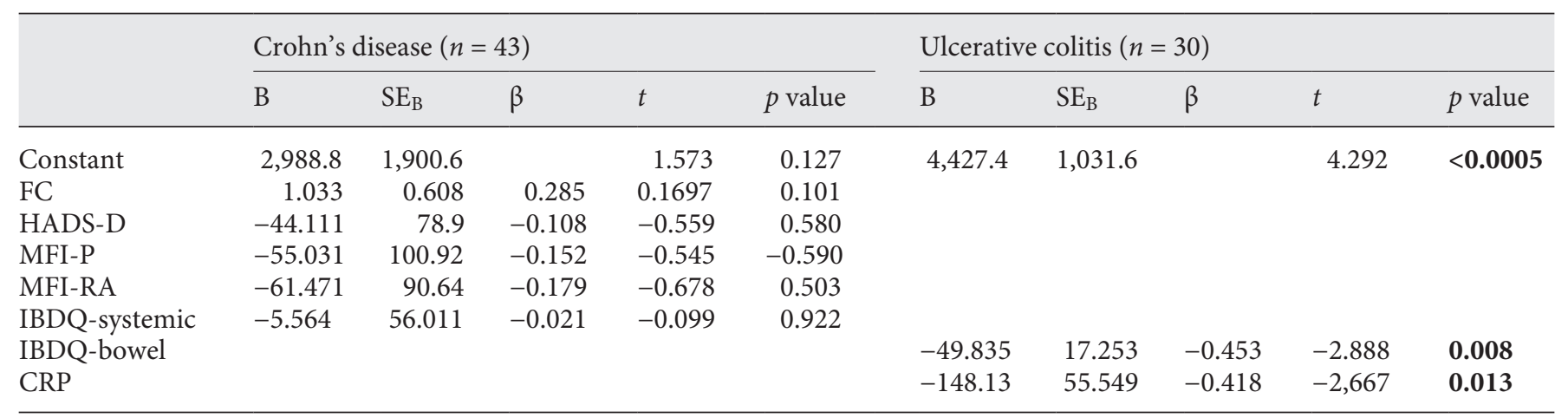

Physical activity is measured as MET-mins/week. IPAQ, International Physical Activity Questionnaire; CRP, C-reactive protein; FC, faecal calprotectin; HADS, Hospital Anxiety and Depression Scale; IBDQ, Inflammatory Bowel Disease Quality of Life Questionnaire; MFI, Multidimensional Fatigue Inventory; MET-mins/week, metabolic equivalent minutes per week.

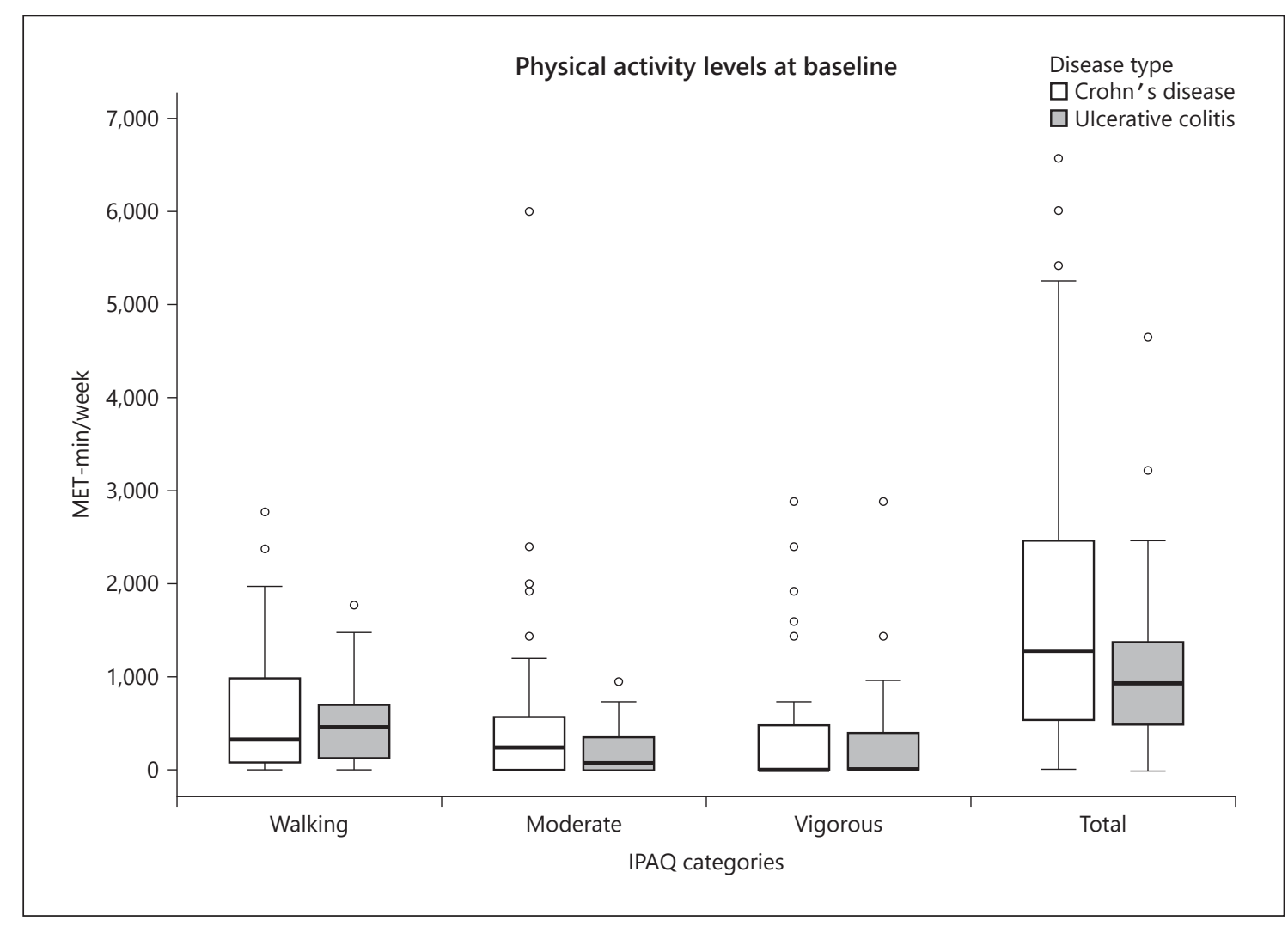

Fig. 2. Physical activity levels of participants (IPAQ categories). IPAQ, International Physical Activity Questionnaire; IBD, inflammatory bowel disease; MET-mins/week, metabolic equivalent minutes per week.

to patients with disease in remission ( 80 vs. $54 \%, p=$ 0.018). Fatigue was the most common barrier reported (34\%), followed by abdominal pain (20\%) and trouble controlling bowels (13\%) (Fig. 3). Participants who felt that their IBD affected their ability to exercise were more likely to have higher fatigue levels (mean difference 3.0; 95\% CI: $1.1-4.9 ; p=0.003$ ) and worse HRQoL across all subsets (IBDQ-total mean difference -19.6; 95\% 


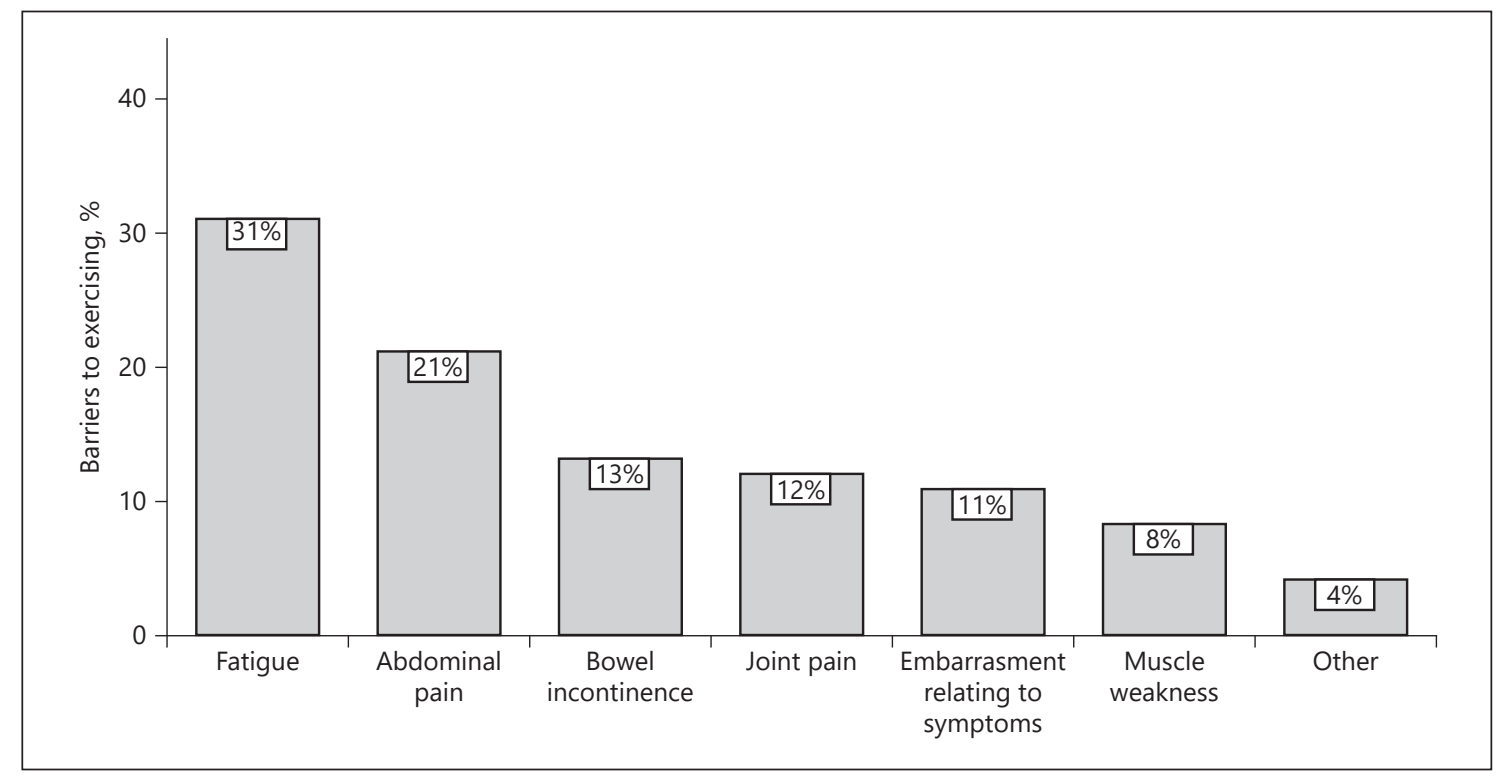

Fig. 3. Barriers identified by participants for engaging in physical activity and exercise, $n=77$.

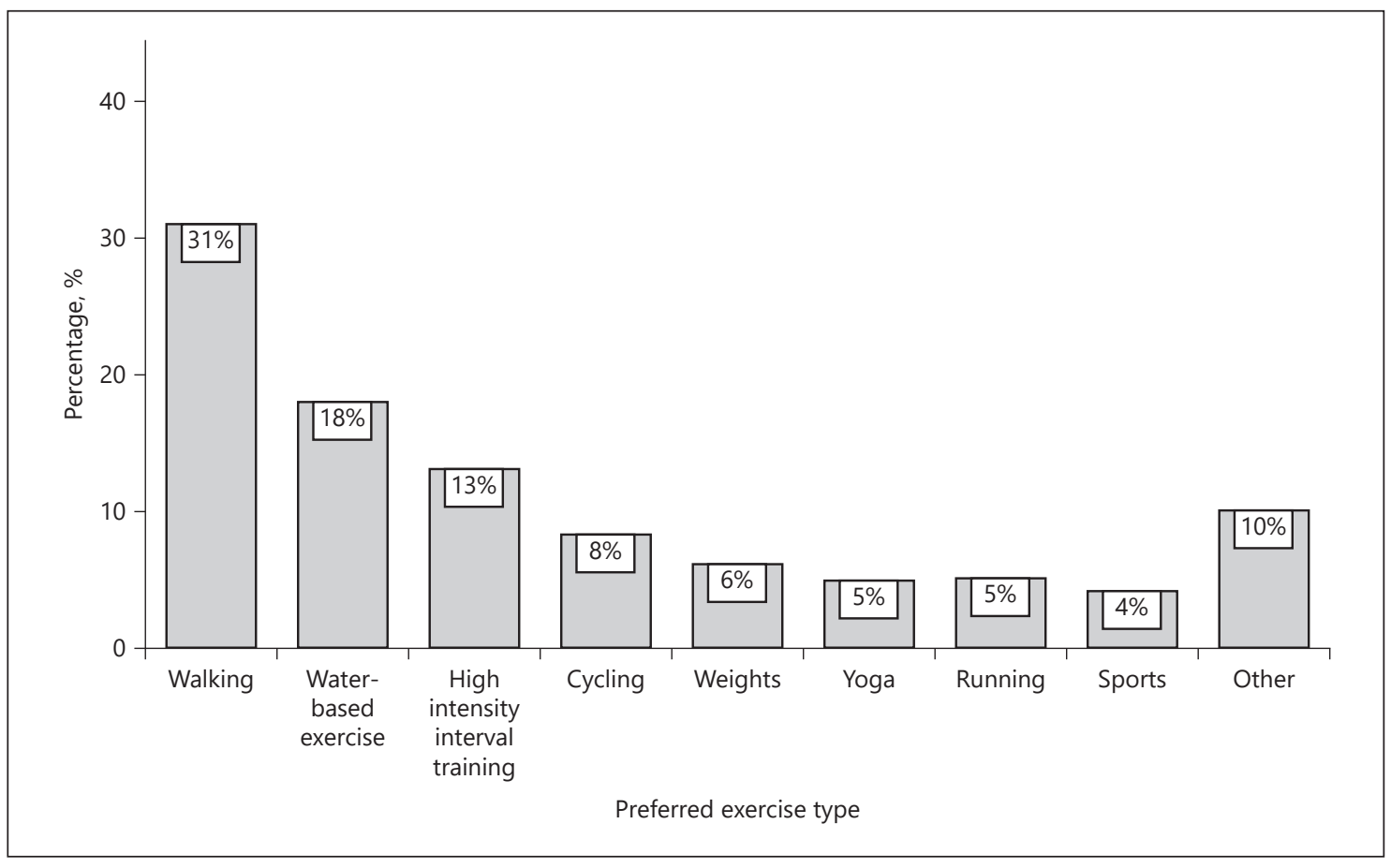

Fig. 4. Most preferred type of exercise for participants, $n=77$.

CI: -31.2 to $-7.9 ; p=0.004)$. The most preferred type of physical activity was walking (30\%), followed by waterbased exercise (20\%) and bodyweight exercises/high-intensity interval training (13\%) (Fig. 4).

\section{Discussion}

This study investigated the current physical activity levels of patients with IBD in Dunedin, New Zealand, and 
further assessed their attitudes and preferences towards exercise. While there is growing knowledge of exercise habits of patients with IBD worldwide, to our knowledge, this study is the first of its kind to be undertaken in New Zealand. Participants' characteristics in this study matched those of previous studies in New Zealand investigating incidence rates of IBD, indicating a reasonably representative group of patients involved $[3,4]$.

\section{Exercise Participation}

Two-thirds of participants in this study met current international guidelines on physical activity [17]. Despite their illness and perceived barriers, participants in this study were more physically active compared to the general population worldwide [16]. While WHO recommend 150 min of moderate physical activity a week at a minimum, for additional health benefits, they also recommend increasing their moderate-intensity aerobic physical activity to $300 \mathrm{~min}$ per week [17]. In addition, there are no specific IBD guidelines of levels of participation in physical activity but significant benefits of exercise are known in patients with IBD [13]. Therefore, even if patients with IBD are meeting minimum physical activity levels, clinicians could aim to encourage patients to increase activity levels to achieve further potential health benefits. This study was a separate analysis of a physical activity intervention study, and therefore it is expected that the majority of participants would be physically active. This phenomenon was also documented by Loudon et al. [30], who showed subjects who volunteer for an exercise study are often more motivated to participate in physical activity than those who choose not to. Therefore, the high level of physical activity in this cohort of patients with IBD is to be anticipated but may limit its generalizability to the wider IBD population.

Although expected, this high level of physical activity contradicts previous literature which indicated patients with IBD participate in less physical activity than the general population worldwide $[19,20]$. Interestingly, studies employing written questionnaires have found similarly unexpectedly high levels of physical activity in patients with IBD $[18,21]$. The IPAQ-short form has reported good reliability [25], but it is also reported to over-estimate physical activity levels by up to $173 \%$ when compared with an accelerometer [31]. A plausible reason for this discrepancy relates to each participant using their own interpretation of what constituted moderate or vigorous physical activity, even when further information was provided, to answer the IPAQ, thus creating a difference between the reported and actual level of physical ac-

Physical Activity Levels in Patients with IBD tivity. Therefore, further studies may need to employ both accelerometers and self-reported questionnaires to fully assess physical activity levels of patients with IBD.

To further investigate what factors may influence physical activity, we investigated the correlation between physical activity levels and physical and psychological well-being. For patients with UC, physical activity levels were independently correlated with HRQoL scores. This aligns with previous research highlighting this relationship [32]. As previously shown in New Zealand [8], in patients with $\mathrm{CD}$, higher fatigue levels were clearly correlated with lower levels of physical activity, similar to previous studies $[8,18]$. When investigating disease activity parameters, only CRP was independently correlated with physical activity levels in patients with UC.

\section{Physical Activity Attitudes and Barriers}

The majority of participants in this current study reported that they felt that their IBD had limited the amount of exercise they can partake in. Other research has reported similar results, indicating that IBD affects perception around exercise capacity and fitness $[18,21]$. Common reasons for the limitation in exercise mentioned in previous studies - fatigue, abdominal pain, and trouble controlling bowels - were prominent for our study participants $[18,21]$. In addition, it is unclear if the levels of reported barriers described here are still evident in patients with IBD who are not as physically active. Further studies could be employed to investigate this and should aim to ensure a cohort of patients with a broad range of physical activity levels is recruited.

Overall, participants with IBD indicated a preference towards low-impact types of exercise, such as walking, as opposed to high-impact types, such as running or playing sports. This result aligns with previous research $[18,21]$. One reason for this may relate to a known extra-intestinal manifestation of IBD - joint arthralgia. Joint arthralgia can make high-impact activities considerably painful and was shown to be a significant barrier to exercise in this study (Fig. 2). Interestingly, participants who felt that their disease activity impacted their ability to exercise had higher levels of fatigue and worse HRQoL than those who did not feel their disease affected their ability to exercise. This may reflect an interplay between physical activity levels, disease barriers, and psychological well-being in patients with IBD which could be further explored.

Unexpectedly, there was a lack of correlation between reported barriers and participation levels in physical activity. Even patients who are more physically active than the expected general population experience high per- 
ceived levels of barriers to exercise. Furthermore, a higher proportion of patients with active disease reported that their IBD limited their ability to exercise when compared to those with disease in remission. However, there was no difference in physical activity participation between the 2 disease severity levels.

In part, this could relate to patients' self-efficacy regarding participation in physical activity. Perhaps patients with active disease have lower self-efficacy in relation to participation in physical activity than those with disease in remission. Indeed, researchers have illustrated that participants with low self-efficacy often have higher perceived barriers to exercise [33]. This itself may result in a thought pattern that they are not capable of participating in high levels of exercise due to their level of active disease and symptoms associated with this, even though they are participating in as much physical activity as those in remission [34]. Indeed, this relationship has been previously reported by Tew et al. [18] who also noted that with increasing disease activity levels, patients' barriers to exercise also increased. Therefore, improving disease activity will have a significant benefit on patient perception of exercise capacity and reduce barriers to physical activity, thereby allowing participants to engage in more physical activity.

\section{Conclusions}

This study indicated that this cohort of patients with IBD in New Zealand participated in higher levels of physical activity than the general population and other IBD populations around the globe, but still experience similarly high levels of barriers to physical activity. In addition, patients with active disease show a clear dissociation between perceived ability to exercise and actual ability to exercise in this cohort. This study adds further evidence of the relationship between physical activity levels and fatigue in patients with IBD. This shows that future studies should be focussing on physical activity as a way of improving the well-being of patients with IBD - especially fatigue, anxiety, and depression. As a clinician, even when engaging with patients who are physically active, our job should be to congratulate, reassure, and reinforce activity levels while also supporting patients to continue to overcome barriers and encourage them to maintain, if not increase, their level of physical activity.

\section{Acknowledgements}

The authors would like to thank Christine Ho, IBD nurse, for her help with patient recruitment. They would also like to thank Marie-Michelle Sullivan, research administrator, for her administrative support during the initiation of the study.

\section{Statement of Ethics}

Participants provided written consent to participate in this study. This study was approved by the Northern B Health and Disability Ethics Committee, New Zealand, Reference No. 17/NTB/44. The trial was registered on ANZCTR, No. ACTRN12617000526370, Universal Trial No. U1111-1192-2859.

\section{Conflict of Interest Statement}

The authors have no conflicts of interest to declare.

\section{Funding Sources}

This work was supported by the Gut Health Network (www. guthealthnetwork.com) of the University of Otago. Georgina Fagan received the Maurice and Phyllis Paykel Trust Research Award.

\section{Author Contributions}

All authors contributed significantly to the conception and design of this study. G.F. was involved in data collection, analysis, and interpretation and initial drafting of the article. H.O. and M.S. were responsible for revising the article for intellectual content. All authors approved the final version of the manuscript.

\section{References}

68
1 Baumgart DC, Carding SR. Inflammatory bowel disease: cause and immunobiology. Lancet. 2007;369(9573):1627-40.

2 Molodecky NA, Soon IS, Rabi DM, Ghali WA, Ferris M, Chernoff G, et al. Increasing incidence and prevalence of the inflammatory bowel diseases with time, based on systematic review. Gastroenterology. 2012;142(1):46e30.
3 Su HY, Gupta V, Day AS, Gearry RB. Rising incidence of inflammatory bowel disease in Canterbury, New Zealand. Inflamm Bowel Dis. 2016;22(9):2238-44.

4 Coppell KJ, Galts CP, Huizing FY, Norton JK, Gray AR, Schultz K, et al. Annual incidence and phenotypic presentation of IBD in southern New Zealand: an 18-year epidemiological analysis. Inflamm Intest Dis. 2018 Nov;3(1):32-9. 
5 Kostic AD, Xavier RJ, Gevers D. The microbiome in inflammatory bowel disease: current status and the future ahead. Gastroenterology. 2014;146(6):1489-99.

6 Czuber-Dochan W, Ream E, Norton C. Review article: description and management of fatigue in inflammatory bowel disease. Aliment Pharmacol Ther. 2013;37(5):505-16.

7 Walker JR, Ediger JP, Graff LA, Greenfeld JM, Clara I, Lix L, et al. The Manitoba IBD cohort study: a population-based study of the prevalence of lifetime and 12-month anxiety and mood disorders. Am J Gastroenterol. 2008; 103(8):1989-97.

8 Aluzaite K, Al-Mandhari R, Osborne $\mathrm{H}$, Ho C, Williams M, Sullivan MM, et al. Detailed multi-dimensional assessment of fatigue in inflammatory bowel disease. Inflamm Intest Dis. 2018;3(4):192-201.

9 Bilski J, Brzozowski B, Mazur-Bialy A, Sliwowski Z, Brzozowski T. The role of physical exercise in inflammatory bowel disease. Biomed Res Int. 2014;2014:429031.

10 Caspersen CJ, Powell KE, Christenson GM Physical activity, exercise, and physical fitness: definitions and distinctions for healthrelated research. Public Health Rep. 1984; 100(2):126-31.

11 Singh MAF. Exercise comes of age: rationale and recommendations for a geriatric exercise prescription. J Gerontol A Biol Sci Med Sci. 2002;57(5):M262-82.

12 Scully D, Kremer J, Meade MM, Graham R, Dudgeon K. Physical exercise and psychological well being: a critical review. Br J Sports Med. 1998;32(2):111-20.

13 Jones PD, Kappelman MD, Martin CF, Chen W, Sandler RS, Long MD. Exercise decreases risk of future active disease in patients with inflammatory bowel disease in remission. Inflamm Bowel Dis. 2015;21(5):1063-71.

14 Benazzato L, Varnier M, Dal Pont E, Larnboglia F, Cardin R, Minotto M, et al. Effect of moderate acute aerobic exercise in patients with Crohn's disease in clinical remission. Dig Liver Dis. 2006;38:S121-22.
15 Klare P, Nigg J, Nold J, Haller B, Krug AB, Mair S, et al. The impact of a ten-week physical exercise program on health-related quality of life in patients with inflammatory bowel disease: a prospective randomized controlled trial. Digestion. 2015;91(3):239-47.

16 Hallal PC, Andersen LB, Bull FC, Guthold R, Haskell W, Ekelund U, et al. Global physical activity levels: surveillance progress, pitfalls, and prospects. Lancet. 2012;380(9838):24757.

17 World Health Organization. Global recommendations on physical activity for health, 18-64 years old. Geneva: World Health Organization; 2011.

18 Tew GA, Jones K, Mikocka-Walus A. Physical activity habits, limitations, and predictors in people with inflammatory bowel disease: a large cross-sectional online survey. Inflamm Bowel Dis. 2016;22(12):2933-42.

19 Van Langenberg DR, Papandony MC, Gibson PR. Sleep and physical activity measured by accelerometry in Crohn's disease. Aliment Pharmacol Ther. 2015;41(10):991-1004.

20 Vogelaar L, Van Den Berg-Emons R, Bussmann H, Rozenberg R, Timman R, Van Der Woude CJ. Physical fitness and physical activity in fatigued and non-fatigued inflammatory bowel disease patients. Scand J Gastroenterol. 2015;50(11):1357-67.

21 DeFilippis EM, Tabani S, Warren RU, Christos PJ, Bosworth BP, Scherl EJ. Exercise and self-reported limitations in patients with inflammatory bowel disease. Dig Dis Sci. 2016; 61(1):215-20.

22 Multi-dimensional approach to increase exercise compliance in patients with inflammatory bowel disease (IBD): a Pilot Study [Internet]. 2017 [cited 2019 Jan 7]. Available from: https: //www.anzctr.org.au/Trial/Registration/ TrialReview. aspx?id=372707\&isReview=true

23 Harvey RF, Bradshaw JM. A simple index of Crohn's-disease activity. Lancet. 1980; 1(8167):514.

24 Walmsley RS, Ayres RC, Pounder RE, Allan RN. A simple clinical colitis activity index. Gut. 1998;43(1):29-32.
25 Craig CL, Marshall AL, Sjöström M, Bauman $\mathrm{AE}$, Booth ML, Ainsworth BE, et al. International physical activity questionnaire: 12 -country reliability and validity. Med Sci Sports Exerc. 2003;35(8):1381-95.

26 Zigmond AS, Snaith RP. The hospital anxiety and depression scale. Acta Psychiatr Scand. 1983.

27 Czuber-Dochan W, Norton C, Bassett P, Berliner S, Bredin F, Darvell M, et al. Development and psychometric testing of inflammatory bowel disease fatigue (IBD-F) patient self-assessment scale. J Crohns Colitis. 2014; 8(11):1398-406.

28 Smets EM, Garssen B, Bonke B, De Haes JC. The multidimensional fatigue inventory (MFI) psychometric qualities of an instrument to assess fatigue. J Psychosom Res. 1995; 39(3):315-25.

29 Irvine EJ, Feagan B, Rochon J, Archambault A, Fedorak RN, Groll A, et al. Quality of life: a valid and reliable measure of therapeutic efficacy in the treatment of inflammatory bowel disease. Gastroenterology. 1994;106(2): 287-96.

30 Loudon CP, Corroll V, Butcher J, Rawsthorne $\mathrm{P}$, Bernstein $\mathrm{CN}$. The effects of physical exercise on patients with Crohn's disease. Am J Gastroenterol. 1999;94(3):697-703.

31 Lee PH, Macfarlane DJ, Lam TH, Stewart SM. Validity of the international physical activity questionnaire short form (IPAQ-SF): a systematic review. Int J Behav Nutr Phys Act. 2011 Oct 21;8(1):115.

32 Taylor K, Scruggs PW, Balemba OB, Wiest MM, Vella CA. Associations between physical activity, resilience, and quality of life in people with inflammatory bowel disease. Eur J Appl Physiol. 2018;118(4):829-36.

33 Stutts WC. Physical activity determinants in adults. Perceived benefits, barriers, and selfefficacy. AAOHN J. 2002;50(11):499-507.

34 Cress ME, Buchner DM, Prohaska T, Rimmer J, Brown M, Macera C, et al. Physical activity programs and behavior counseling in older adult populations. Med Sci Sports Exerc. 2004;36(11):1997-2003. 